\title{
Effect of Quadriceps Strength and Proprioception on Risk for Knee Osteoarthritis
}

\author{
NEIL A. SEGAL ${ }^{1}$, NATALIE A. GLASS ${ }^{1}$, DAVID T. FELSON ${ }^{2}$, MICHAEL HURLEY ${ }^{3}$, MEI YANG ${ }^{2}$, \\ MICHAEL NEVITT $^{4}$, CORA E. LEWIS ${ }^{5}$, and JAMES C. TORNER ${ }^{1}$ \\ ${ }^{I}$ The University of Iowa, Iowa City, IA; ${ }^{2}$ Boston University, Boston, MA; ${ }^{3}$ King's College London, London, England, \\ UNITED KINGDOM; ${ }^{4}$ University of California at San Francisco, San Francisco, CA; and ${ }^{5}$ University of Alabama \\ at Birmingham, Birmingham, $A L$
}

\begin{abstract}
SEGAL, N. A., N. A. GLASS, D. T. FELSON, M. HURLEY, M. YANG, M. NEVITT, C. E. LEWIS, and J. C. TORNER. Effect of Quadriceps Strength and Proprioception on Risk for Knee Osteoarthritis. Med. Sci. Sports Exerc., Vol. 42, No. 11, pp. 2081-2088, 2010. Purpose: Impaired quadriceps strength and joint position sense (JPS) have been linked with knee osteoarthritis (OA) crosssectionally. Although neither has been independently associated with incident radiographic OA, their combination may mediate risk. The purpose of this study was to determine whether better sensorimotor function protects against the development of incident radiographic or symptomatic knee OA. Methods: The Multicenter Osteoarthritis study is a longitudinal study of adults aged 50-79 yr at high risk for knee OA. Participants underwent bilateral, weight-bearing, fixed-flexion radiographs, JPS acuity tests, and isokinetic quadriceps strength tests. The relationships between combinations of the tertiles of sex-specific baseline peak strength and mean JPS and development of incident radiographic (Kellgren-Lawrence (KL) grade $\geq 2$ ) or symptomatic knee OA (KL grade $\geq 2$ and frequent knee pain or stiffness) at a 30-month follow-up were evaluated. Secondary analyses defined JPS as the variance during the 10 JPS trials and also assessed the interaction of strength and JPS in predicting each outcome. Results: The study of incident radiographic knee OA included 1390 participants ( $\mathrm{age}=61.2 \pm 7.9 \mathrm{yr}$ and body mass index $=29.4 \pm 5.1 \mathrm{~kg} \cdot \mathrm{m}^{-2}$ ), and the study of incident symptomatic knee OA included 1829 participants (age $=62.2 \pm 8.0 \mathrm{yr}$ and body mass index $=30.0 \pm 5.4 \mathrm{~kg} \cdot \mathrm{m}^{-2}$ ). Greater strength at baseline protected against incident symptomatic but not radiographic knee OA regardless of JPS tertile. There was no significant relationship between the strength-JPS interaction and the development of radiographic or symptomatic knee OA. Conclusions: The finding that quadriceps strength protected against incident symptomatic but not radiographic knee OA regardless of JPS tertile suggests that strength may be more important than JPS in mediating risk for knee OA. Key Words: KNEE PAIN, REHABILITATION, EPIDEMIOLOGY, MUSCULOSKELETAL
\end{abstract}

$\mathrm{T}$ The etiology of osteoarthritis (OA) is both biomechanical and biochemical, and there is no known cure, making effective rehabilitation particularly important. The knee is the weight-bearing joint most commonly affected by $\mathrm{OA}$ and is second in overall OA incidence (5). Compared with those without knee OA, those with knee OA demonstrate slower performance and increased need for functional modifications (25). This is particularly the case for those with reduced lower limb sensorimotor function. Therefore, there is a need to better

\footnotetext{
Address for correspondence: Neil A. Segal, M.D., M.S., Departments of Orthopaedics and Rehabilitation, Radiology, and Epidemiology, University of Iowa, 200 Hawkins Dr., 0728 JPP, Iowa City, IA 52242-1088; E-mail: segal-research@uiowa.edu.

Submitted for publication January 2010.

Accepted for publication March 2010.

0195-9131/10/4211-2081/0

MEDICINE \& SCIENCE IN SPORTS \& EXERCISE $_{\circledast}$ Copyright $(C 2010$ by the American College of Sports Medicine

DOI: 10.1249/MSS.0b013e3181dd902e
}

understand potentially modifiable risk factors for development of knee OA and disablement.

Impaired quadriceps strength and proprioception have been linked cross-sectionally with knee OA $(9-11,13,17,23,38,39)$. Recently, we found increased knee extensor strength decreased risk of developing symptomatic knee OA in women observed for 30 months (35). However, we did not find a relationship between knee extensor strength and incident radiographic knee OA in that study. Likewise, impaired proprioception has not been associated with structural damage at the knee longitudinally (6). Similar to other pathological processes, more than one predisposing factor may be required to lead to disease. It is currently unknown whether better sensorimotor function, defined as the combination of higher quadriceps strength and better proprioception, protects against development of incident radiographic or symptomatic knee OA.

By definition, stability at the knee joint requires internal forces of sufficient magnitude to counteract external forces acting at the knee. The quadriceps muscle absorbs loads and provides dynamic stability. Weakness of the quadriceps may alter local contact stress in a manner detrimental to 
articular cartilage (14). It may also lead to increased impulse loading, which has been associated with knee pain and may contribute to knee OA $(20,33)$. In a study of resistance training in women, heavier women who did not strength train had significantly higher rates of loading during gait compared with those who did (29). These results suggest that if excess loading is predictive of OA, increased quadriceps strength may protect the knee. Despite the contribution of the quadriceps to shock absorption and stability at the knee during gait, there has been only a single longitudinal study that has linked quadriceps weakness with incident radiographic knee OA (40). This finding was present only in women and was not reproduced in a more recent study (35). Thus, additional factors such as those that affect muscle coordination during gait may play a significant role in the development of radiographic knee OA and should be examined longitudinally.

Proprioception, of which joint position sense (JPS) is a component, contributes to dynamic knee joint stability by coordinating the actions of the quadriceps, hamstrings, and associated muscles. Signals from muscle spindles are important for midrange perception of joint angle and joint mechanoreceptors, such as pacinian corpuscles, Ruffini end organs, and Golgi joint and tendon receptors in other knee joint components (tendons, joint capsule, cruciate and collateral ligaments, and meniscal attachments) are important for detecting stretch at extremes of joint range of motion $(13,36,41)$. Older adults with symptomatic knee OA have been reported to have impaired proprioception, strength, and balance, as well as fewer mechanoreceptors in ligaments, and a higher rate of falls compared with those without symptomatic knee OA (13). Data, which show that proprioception decreases with age $(3,30)$ and that reduced proprioception is associated cross-sectionally with knee OA $(10,11,26,30,36,38)$, suggest that impaired proprioception may contribute to the onset or progression of knee OA. Additional evidence for the contribution of impaired proprioception to the pathogenesis of knee OA are results of cross-sectional studies of individuals with unilateral knee OA that have shown proprioception is impaired in both the OA involved and uninvolved knee. Results from a recent longitudinal study did not demonstrate a relationship between greater error in knee joint reposition sense and development of knee structural damage (6). Therefore, it is possible that results from cross-sectional studies reflect that knee OA contributes to impaired proprioception rather than the other way around. However, further longitudinal studies are needed to better characterize the temporal relationships.

We are unaware of any previous longitudinal studies of the effects of the combination of knee extensor strength and JPS on the development of knee OA. Because a combination of sensorimotor factors may be necessary to mediate risk, this analysis was designed to determine whether the combination of high knee extensor strength and knee JPS protects against the development of incident radiographic or incident symptomatic knee OA 30 months later.

\section{METHODS}

This study enrolled individuals from the Multicenter Osteoarthritis (MOST) study, a longitudinal cohort study of risk factors for knee OA. MOST is composed of 3026 community-dwelling men and women aged 50-79 yr with knee OA or known risk factors for knee OA. Enrollment has been described previously (35). Briefly, recruitment methods included mass mailings and advertisements and eligibility screening by telephone for known knee OA risk factors including knee injury or surgery or obesity. Potential participants were excluded for a history of (or planned) bilateral knee replacement, nonresected cancer (with the exception of non-melanoma skin cancer), history of chemotherapy or radiation therapy, history of rheumatologic disease, or plan to move out of the area within $3 \mathrm{yr}$ of enrollment. This study was approved by the investigators' institutional review boards. All participants provided written informed consent using an institutional review board-approved consent process.

In this study, the knee was the basic unit for analyses. Knees were excluded if, when attempting the knee extensor strength test, participants reported pain that prevented them from completing the test. For the study of incident radiographic knee OA (Kellgren-Lawrence (KL) grade $\geq 2$ ), eligible participants were those who did not have knee OA $(\mathrm{KL}$ grade $<2)$ at baseline. For the study of incident symptomatic knee OA, eligible participants did not have the combination of knee OA and frequent knee symptoms at baseline.

\section{Measurements}

Body mass index. Height in centimeters (Stadiometer; Holtain, Wales, UK) and weight in kilograms (balance beam scale) were measured by trained and certified staff, and body mass index (BMI; $\mathrm{kg} \cdot \mathrm{m}^{-2}$ ) was calculated.

Knee extensor strength measurements. Concentric knee extensor strength was assessed with a Cybex 350 computerized isokinetic dynamometer (Avocent, Huntsville, $\mathrm{AL}$ ) at $60^{\circ} \cdot \mathrm{s}^{-1}$ through a range of motion of approximately $90^{\circ}$, and a chair back angle of $85^{\circ}$. HUMAC software version 4.3.2/Cybex 300 for Windows 98 Software Package (CSMi, Stoughton, MA) was used for data acquisition. Participants were provided instructions using a standardized script. After three practice trials using 50\% perceived effort, four repetitions were completed for extensor torque while using a standardized script to verbally encourage maximum effort. Participants' concentric knee extensor strength $(\mathrm{N} \cdot \mathrm{m})$ was considered the peak torque obtained during four trials.

Participants were stabilized in the seat with a seatbelt over the lap and torso. Each of the two centers had a single Cybex machine. Within each center, the same Cybex machine was used on all participants. Both centers used the same model of computerized isokinetic dynamometer and the same software as well as the same weights for weekly and monthly calibration, using the same protocol. The preload force was standardized among participants with 
differing shank lengths by adjusting the dynamometer arm length and the position of the Johnson Antishear Accessory to each participant.

Trained examiners, certified in the standardized MOST strength testing protocol, underwent annual recertification to ensure uniformity of the strength testing protocol. Examiners calibrated the isokinetic dynamometer position, angular velocity and torque (at 25 and $245 \mathrm{~N} \cdot \mathrm{m}$ ) monthly. The strength testing protocol had a test-retest reliability (intraclass correlation coefficient) of $0.94(0.82-0.99)$, a coefficient of variation of $8 \%(6 \%-12 \%)$ and a within-subject variation of $6.3 \mathrm{~N} \cdot \mathrm{m}(4.71-9.63 \mathrm{~N} \cdot \mathrm{m})$. After the strength test, participants were asked whether they experienced pain during the test. If the response was affirmative, the participant was then asked "Did this pain prevent you from pushing or pulling as hard as you can?" Participants who responded affirmatively to this second specific question were excluded because of the inability to obtain a reliable strength measurement.

JPS. JPS was measured in 2481 of 3026 subjects in the right leg. Participants were given two practice trials and then were asked to reproduce 10 different knee flexion angles determined using an electrogoniometer (Model SG150 twin-axis goniometer; Biometric Ltd., Ladysmith, VA) and a protocol described previously $(6,16)$. Briefly, the participant was seated in a chair, slowly extended the leg to a set angle (active JPS), and was asked to hold that position for $5 \mathrm{~s}$ before slowly relaxing the leg to the freely dangling position. The participant was then asked to reproduce that angle without being able to see his/her leg. The difference between the test angle and the reproduced angle was recorded. Participants were asked to reproduce angles between $35^{\circ}-45^{\circ}, 15^{\circ}-25^{\circ},>45^{\circ}, 5^{\circ}-15^{\circ}, 25^{\circ}-35^{\circ}, 15^{\circ}-25^{\circ}$, $35^{\circ}-45^{\circ}, 5^{\circ}-15^{\circ},>45^{\circ}$, and $25^{\circ}-35^{\circ}$ in the same preset order. The electrogoniometer was attached to the lateral aspect of the right leg, across the knee joint. The electrogoniometer accuracy was tested daily against a handheld goniometer. Knees without at least $50^{\circ}$ of movement between straight and $90^{\circ}$ flexion were not tested.

JPS was operationally defined as the average of the absolute value of the difference (i.e., error) between the 10 preset and participant-reproduced knee flexion angles. For naming the categories of JPS, "high" was used for the tertile with the lowest raw JPS scores (i.e., the least joint reposition error), indicating high proprioceptive acuity. The variance between the 10 preset and participant-reproduced knee flexion angles was also evaluated. The variance is a measure of the difference between the mean error and the absolute value of the error for each reproduced angle. In other words, the variance is equal to the sum of the squares of the deviation from the mean value for each limb.

Radiographic knee OA assessment. Weight-bearing, fixed-flexion posteroanterior (31) and lateral radiographs (24) of the knees were obtained at baseline and after 30 months according to the MOST radiograph protocol as previously described $(6,34)$. Radiographs of the contralateral knee in participants with unilateral knee replacement were taken. Each participant's baseline and follow-up radiographs were paired and scored by two independent readers (an experienced academically based musculoskeletal radiologist and a rheumatologist experienced in study reading) according to the KL scale (22). As noted in previous articles $(6,34)$, incident radiographic disease was designated only if there was a KL grade $\geq 2$ at the 30 -month follow-up in a knee with $\mathrm{KL}$ grade $<2$ at baseline. Readers were not blinded to radiograph sequence but were blinded to both subject strength and JPS, the predictors in this study. For cases where the two readers disagreed on the presence of incident radiographic tibiofemoral OA, an adjudication panel of three experienced readers decided. We defined incident $\mathrm{OA}$ as new onset tibiofemoral KL grade $\geq 2$ or new onset features of patellofemoral OA (7).

Knee symptoms. At both the baseline assessment and at the 30-month follow-up assessment, participants were asked about the presence of knee symptoms in each knee twice - on a telephone interview and at a clinic visit. During the telephone interviews, trained and certified interviewers asked participants: "During the past $30 \mathrm{~d}$, have you had pain, aching or stiffness in or around your knee on most days?" Knee symptoms were assessed again at the baseline clinic visit, where participants were asked the same question again about knee pain, aching, or stiffness. There was a median of $34 \mathrm{~d}$ between the telephone interview and the baseline clinic visit. Participants who responded negatively on either the telephone interview or the baseline visit questionnaire were considered to not have frequent knee symptoms at baseline. At the 30-month telephone screen and clinic visit, participants were asked again the same question regarding pain, aching, or stiffness in each knee on most of the past $30 \mathrm{~d}$. Incident knee symptoms were defined by an affirmative response on both the telephone interview and clinic visit at 30 months when both had not been affirmative at baseline.

Physical activity and medical history. At baseline, participants completed the Physical Activity Scale for the Elderly (PASE: New England Research Institute, Watertown, MA) questionnaire, and activity scores were calculated (43). Participants were also asked whether they had suffered a knee injury or underwent a knee surgery.

\section{Outcomes}

Incident radiographic knee OA. Knees met criteria for incident radiographic whole knee OA if they had no radiographic tibiofemoral or patellofemoral OA at baseline (KL grade $<2$ ) and had radiographic tibiofemoral or patellofemoral OA (KL grade of $\geq 2$ ) on 30 -month radiographs.

Incident symptomatic knee OA. At the baseline and 30-month visits, we obtained radiographs and asked during phone and clinic interviews about the presence of knee pain or stiffness on most days of the past month. 
Incident symptomatic whole knee OA was defined as the combination of frequent knee symptoms and radiographic $\mathrm{OA}$ in the tibiofemoral or patellofemoral compartments (whole knee OA) at the 30-month follow-up but not at the baseline visit. Recognizing that OA symptoms fluctuate (21), we felt that at follow-up subjects needed to answer "yes" to the knee symptom questions on both telephone and clinic interviews.

Therefore, knees met criteria for incident symptomatic whole knee (tibiofemoral or patellofemoral) OA if they met one of the following four definitions:

1) at baseline, they did not have radiographic whole knee OA (x-ray-negative) and had daily knee symptoms, but at 30 months, they had the combination of radiographic whole knee OA (x-ray-positive) and knee symptoms on both the screen and clinic visits as described above (symptoms positive); or if

2) at baseline, they had radiographic whole knee OA (x-ray-positive) but did not have symptoms on both the screen and clinic visits (symptoms-negative on one or both interviews), but at 30 months, they consistently had frequent knee symptoms (symptoms-positive on both interviews); or if

3) at baseline, they had neither radiographic whole knee OA (x-ray-positive) nor symptoms but developed both at 30 months; or if

4) they did not have both radiographic whole knee OA and symptoms at baseline (x-ray- and/or symptomsnegative) and underwent knee arthroplasty between baseline and follow-up as treatment for OA.

Statistical methods. Data were examined by first calculating univariate distributions for age, BMI, physical activity score (PASE), frequency of knee OA diagnosis (categorical KL grade $\geq 2$ vs KL grade $<2$ ), history of knee surgery, history of knee injury, and sex for eligible and ineligible subjects. Distributions were compared with differences between those eligible for the study and those ineligible because of missing data. We then analyzed the associations among peak strength, JPS (average and variance), and covariates listed above. Combined tertiles were determined in a sex-specific manner (i.e., low strength for women was low strength compared with strength in other women and low strength for men was low in comparison with other men). JPS data for the right lower limb were used in combination with strength data for the right and left knee extensor muscles because JPS is known to be similar bilaterally even in people with unilateral knee OA $(10,28)$. A separate set of analyses was completed for only right lower limb JPS and strength data to confirm that this assumption did not alter the results.

SAS software version 9.1 (SAS Institute, Inc., Cary, NC) was used to complete multivariable logistic regression, with the dependent variable being either incident radiographic knee OA or incident symptomatic knee OA and the independent variable being homologous tertiles of strength and JPS, using the group with the lowest peak knee extensor strength and the worst JPS score as the reference group. Analyses adjusted for the effects of age, BMI, PASE score, and history of knee surgery or knee injury at baseline. These analyses were completed using homologous tertiles of strength and mean JPS as well as strength and variance in JPS. Results were expressed as odds ratios (OR) and 95\% confidence intervals (CI). Analyses were repeated treating both baseline strength and JPS as continuous measures and assessing each of these as well as their interaction in predicting each of the outcomes at follow-up. Analyses of outcomes were lower limb-based, and generalized estimating equations were used in all models to adjust for correlations between limbs and visits within each subject. For the study of incident radiographic knee OA, 2276 limbs provided sufficient statistical power $(>80 \%)$ to detect a difference in OR of 0.22 between those in any of the other categories versus those in the low/low (referent) category. For the study on incident symptomatic knee OA, 3166 limbs provided sufficient statistical power $(>80 \%)$ to detect a difference in OR of 0.49 between those in any of the other categories versus those in the low/low (referent) category.

\section{RESULTS}

Baseline characteristics for the studies of incident radiographic whole knee OA and incident symptomatic knee OA are presented in Table 1. Sex-specific tertiles of strength and JPS were determined separately then combined for analyses. The means for each sex-specific strength and JPS tertile are shown in Table 2.

Incident radiographic whole knee OA. Of 5331 knees, 2276 (58.2\% women) met eligibility criteria, whereas 2479 knees with OA at baseline and 576 knees with inflammatory arthritis, missing strength or JPS data, or report of pain that prevented the strength measure at baseline were excluded (Fig. 1). For eligible participants, the mean age was $61.2 \pm 7.9 \mathrm{yr}$ and mean BMI was $29.4 \pm$ $5.1 \mathrm{~kg} \cdot \mathrm{m}^{-2}$. We found that baseline mean JPS correlated

TABLE 1. Baseline characteristics.

\begin{tabular}{|c|c|c|c|c|c|c|c|}
\hline & Age (yr) & BMI $\left(\mathrm{kg} \cdot \mathrm{m}^{-2}\right)$ & Knee Extensor Strength (N.m) & Sex & PASE Score & JPS Mean $\left({ }^{\circ}\right)$ & JPS Variance $\left({ }^{\circ}\right)$ \\
\hline $\begin{array}{l}\text { Study of incident radiographic knee } 0 A \\
\qquad(n=1390 \text { subjects/2276 knees })\end{array}$ & $61.2 \pm 7.9$ & $29.4 \pm 5.1$ & $96.4 \pm 43.6$ & $\begin{array}{l}588 \text { men } \\
802 \text { women }\end{array}$ & $186.6 \pm 88.6$ & $4.2 \pm 1.8$ & $7.6 \pm 6.0$ \\
\hline $\begin{array}{l}\text { Study of incident symptomatic knee OA } \\
\qquad(n=1829 \text { subjects/3166 knees })\end{array}$ & $62.2 \pm 8.0$ & $30.0 \pm 5.4$ & $94.1 \pm 43.1$ & $\begin{array}{c}748 \text { men } \\
1081 \text { women }\end{array}$ & $181.5 \pm 88.7$ & $4.2 \pm 1.8$ & $7.9 \pm 6.2$ \\
\hline
\end{tabular}

Mean \pm SD 


\begin{tabular}{|c|c|c|c|c|c|}
\hline \multirow[b]{2}{*}{ Study } & \multirow[b]{2}{*}{ Tertile } & \multicolumn{2}{|c|}{$\begin{array}{l}\text { Sex-Specific Tertiles of Knee Extensor } \\
\text { Strength (N.m) }\end{array}$} & \multicolumn{2}{|c|}{ Sex-Specific Tertiles of Mean JPS $\left(^{\circ}\right.$} \\
\hline & & Male & Female & Male & Female \\
\hline \multirow[t]{6}{*}{ Incident radiographic knee $\mathrm{OA}$} & Highest & $176.27 \pm 25.78$ & $100.34 \pm 17.25$ & $2.40 \pm 0.52$ & $2.52 \pm 0.58$ \\
\hline & & $n=313$ & $n=451$ & $n=328$ & $n=446$ \\
\hline & Middle & $127.58 \pm 9.79$ & $71.21 \pm 5.91$ & $3.93 \pm 0.40$ & $3.95 \pm 0.38$ \\
\hline & & $n=328$ & $n=429$ & $n=306$ & $n=439$ \\
\hline & Lowest & $85.14 \pm 18.76$ & $45.47 \pm 10.39$ & $6.26 \pm 1.45$ & $6.23 \pm 1.43$ \\
\hline & & $n=311$ & $n=444$ & $n=318$ & $n=439$ \\
\hline \multirow[t]{6}{*}{ Incident symptomatic knee $0 \mathrm{~A}$} & Highest & $172.83 \pm 26.00$ & $98.88 \pm 16.60$ & $2.38 \pm 0.53$ & $2.51 \pm 0.58$ \\
\hline & & $n=441$ & $n=611$ & $n=432$ & $n=631$ \\
\hline & Middle & $125.17 \pm 9.59$ & $69.30 \pm 6.26$ & $4.01 \pm 0.45$ & $3.98 \pm 0.39$ \\
\hline & & $n=440$ & $n=620$ & $n=449$ & $n=616$ \\
\hline & Lowest & $82.53 \pm 18.54$ & $44.47 \pm 9.68$ & $6.36 \pm 1.39$ & $6.33 \pm 1.42$ \\
\hline & & $n=429$ & $n=625$ & $n=429$ & $n=609$ \\
\hline
\end{tabular}

Mean \pm SD

with age $(r=0.09, P=0.003)$ and knee pain $(r=0.08$, $P=0.01)$, whereas JPS variance correlated only with knee pain $(r=0.08, P=0.01)$.

Results of the analyses adjusted for sex, age, BMI, history of knee injury, history of knee surgery, and physical activity at baseline showed no relationship between combinations of knee extensor strength and mean JPS and incident radiographic knee OA (Table 3). High knee extensor strength and high (best) mean JPS were not protective against incident radiographic knee OA when evaluated individually. Further, when examined as continuous variables, the interaction between knee extensor strength and mean JPS was not associated with incident radiographic knee OA $(P=0.4697$ in men and $P=0.9306$ in women $)$. In all analyses, only BMI consistently predicted the development of incident radiographic knee OA (all $P<0.0001$ ).

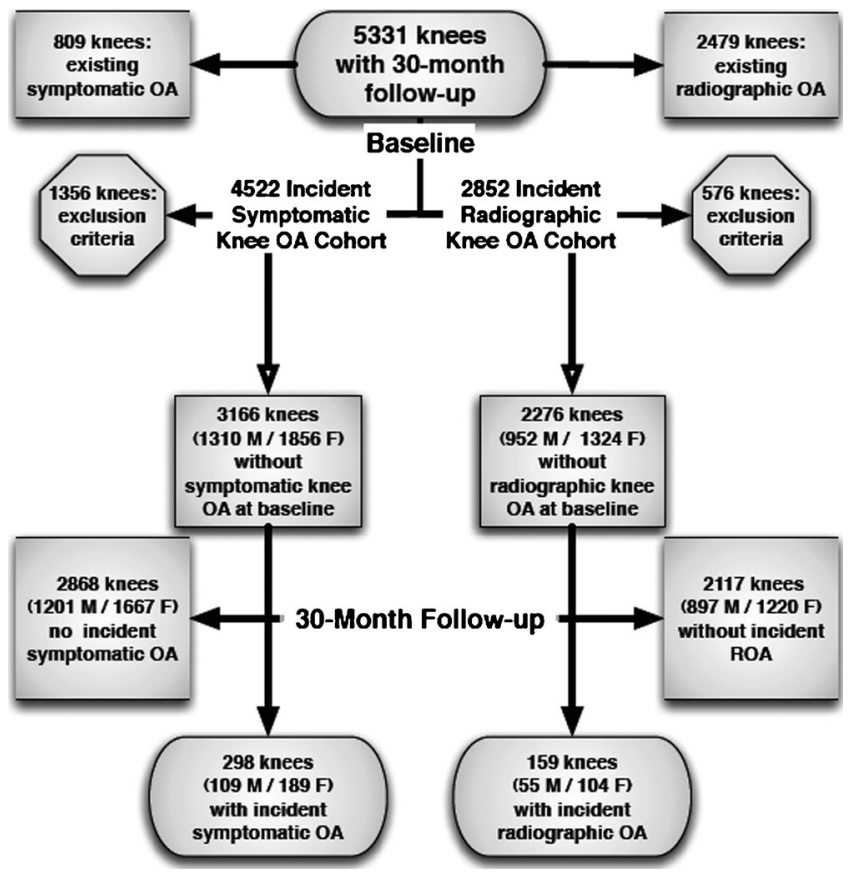

FIGURE 1-Subject inclusion diagram.
Incident symptomatic whole knee OA. A total of 809 knees with symptomatic knee OA present at baseline as well as 1356 knees with inflammatory arthritis, missing data, or report of pain that prevented the strength test at baseline were excluded. A total of 3166 knees $(58.6 \%$ women) met eligibility criteria and were included in this study. The mean age and BMI at baseline was $62.2 \pm 8.0 \mathrm{yr}$ and $30.0 \pm 5.4 \mathrm{~kg} \cdot \mathrm{m}^{-2}$, respectively. Baseline mean JPS and JPS variance scores were worse with older age $(r=0.11$, $P<0.0001 ; r=0.09, P=0.0004)$, lower physical activity level $(r=-0.06, P=0.01 ; r=-0.06, P=0.02)$, and higher pain scores $(r=0.07, P=0.005 ; r=0.09, P=0.0003)$.

A total of 298 knees developed incident symptomatic knee OA (definition 1, $n=21$; definition 2, $n=214$; definition $3, n=25$; definition $4, n=38$ ). We found a decrease in risk for incident symptomatic knee OA in subjects with greater knee extensor strength compared with those with lower knee extensor strength regardless of JPS in analyses adjusted for age, BMI, knee injury, knee surgery, physical activity, and sex (Table 4). When examined separately, high compared with low knee extensor strength was associated with a decreased risk for incident symptomatic knee OA $(\mathrm{OR}=0.43,95 \% \mathrm{CI}=0.31-0.61, P<0.0001)$. However, no relationship was found between mean JPS and incident symptomatic knee OA. Results from analyses using JPS variance scores rather than mean JPS were similar (results not shown). There were significant relationships among age, BMI, and history of surgery and incident symptomatic

TABLE 3. The effect of baseline knee extensor strength and mean JPS on incident radiographic knee $0 A$.

\begin{tabular}{lcc}
\hline $\begin{array}{l}\text { Knee Extensor Strength } \\
\text { and JPS Categories } \\
\text { (High/Middle/Low) }\end{array}$ & $\begin{array}{c}\text { Cases/Total } \\
\boldsymbol{n}(\%)\end{array}$ & $\begin{array}{c}\text { OR (95\% CI) for Outcome, Adjusted } \\
\text { for Baseline Age, BMI, Knee Injury, } \\
\text { Knee Surgery, PASE, and Sex }\end{array}$ \\
\hline High/high & $20 / 278(7.2)$ & $0.91(0.44-1.89)$ \\
High/middle & $12 / 242(5.0)$ & $0.57(0.25-1.30)$ \\
High/low & $17 / 244(7.0)$ & $1.02(0.48-2.17)$ \\
Middle/high & $14 / 244(5.7)$ & $0.79(0.37-1.71)$ \\
Middle/middle & $17 / 266(6.4)$ & $0.87(0.42-1.81)$ \\
Middle/low & $18 / 247(7.3)$ & $1.03(0.51-2.08)$ \\
Low/high & $17 / 252(6.8)$ & $0.96(0.48-1.95)$ \\
Low/middle & $26 / 237(11.0)$ & $1.60(0.82-3.11)$ \\
Low/low & $18 / 266(6.8)$ & Referent \\
\hline
\end{tabular}


TABLE 4. The effect of baseline knee extensor strength and mean JPS on incident symptomatic knee $O A$.

\begin{tabular}{lcc}
\hline $\begin{array}{l}\text { Knee Extensor } \\
\text { Strength and JPS } \\
\text { Categories } \\
\text { (High/Middle/Low) }\end{array}$ & $\begin{array}{c}\text { Cases/Total } \\
\boldsymbol{n}(\%)\end{array}$ & $\begin{array}{r}\text { OR (95\% CI) For Outcome, } \\
\text { Adjusted for Baseline Age, BMI, Knee } \\
\text { Injury, Knee Surgery, PASE, and Sex }\end{array}$ \\
\hline High/high & $26 / 388(6.7)$ & $0.42(0.25-0.72)^{a}$ \\
High/middle & $21 / 344(6.1)$ & $0.36(0.20-0.66)^{a}$ \\
High/low & $21 / 320(6.6)$ & $0.46(0.26-0.82)^{a}$ \\
Middle/high & $27 / 331(8.2)$ & $0.53(0.32-0.89)^{a}$ \\
Middle/middle & $20 / 384(5.2)$ & $0.37(0.22-0.63)^{a}$ \\
Middle/low & $29 / 345(8.4)$ & $0.50(0.29-0.86)^{a}$ \\
Low/high & $47 / 344(13.7)$ & $0.87(0.55-1.38)$ \\
Low/middle & $52 / 337(15.4)$ & $1.01(0.64-1.58)$ \\
Low/low & $55 / 373(14.8)$ & Referent \\
\hline
\end{tabular}

${ }^{a}$ Significant predictor of incident symptomatic knee $0 \mathrm{~A}$.

knee $\mathrm{OA}$ in all analyses (all $P<0.05$ ). In continuous analyses, the interaction between knee extensor strength and JPS did not predict incident symptomatic knee OA $(P=0.8482$ in men and $P=0.3657$ in women $)$.

\section{DISCUSSION}

Results of this study show that greater knee extensor strength was associated with a decrease in risk for incident symptomatic but not incident radiographic knee OA regardless of JPS tertile. These results confirm previous findings in the MOST cohort regarding knee extensor strength (35) and are consistent with findings regarding JPS and knee joint structural change (6).

Although we are aware of no longitudinal studies that evaluated whether a combination of quadriceps strength and proprioceptive acuity reduced risk of developing knee OA with which to compare these results, there have been crosssectional studies evaluating each of these relationships separately in individuals with preexisting knee OA $(13,30)$. Hassan et al. (12) found that individuals with symptomatic knee OA have weaker quadriceps, reduced knee joint proprioception, and increased postural sway compared with age- and sex-matched controls. In that study, knee joint proprioception was measured as the ability to actively reproduce a passively positioned test angle, and the maximal voluntary contraction of the quadriceps was measured isometrically. In a more recent study, Mohammadi et al. (30) evaluated dynamic balance and, using similar measures of JPS and quadriceps strength to those used in prior studies, found that women with symptomatic knee OA had reduced dynamic balance, strength, and JPS compared with women without symptomatic knee OA. Both studies made comparisons with control subjects without knee symptoms. Hall et al. (11) evaluated individuals with and without knee pain and radiographic knee $\mathrm{OA}$ and found that those with symptomatic knee OA had greater weakness, self-reported disability, and postural sway compared with those with isolated knee pain, isolated radiographic knee OA, or neither. However, using the same methods to measure JPS as Hassan, there were no significant differences in proprioceptive acuity between any of these groups.
We hypothesized that sensorimotor dysfunction may place the knee joint at risk of osteoarthritic damage. The quadriceps muscle is the principal dynamic stabilizer of the knee joint, and muscle spindles provide a substantial contribution to proprioceptive acuity. Thus, impaired proprioceptive acuity and quadriceps muscle weakness could leave the knee joint vulnerable to initial and further injury. To investigate this possibility, we estimated proprioceptive acuity by measuring knee joint reposition error. In evaluating both the mean and variance of 10 trials, we found that strength, regardless of JPS score, protected against incident symptomatic knee OA. This would suggest that impaired proprioceptive acuity, as measured in this study, does not increase risk of radiographic or symptomatic knee OA within the 30-month time frame.

Proprioception is defined as sense of joint motion (kinesthesia) as well as JPS. Accordingly, there are several components of proprioception, and different aspects can be measured in seated or weight-bearing positions and through passive or active tests. In studies of OA, proprioception of the knee joint has been measured as joint motion sense in some studies $(23,32,38,42)$ and JPS in others $(1,6,10$, 19,30). An important difference in the proprioception measures used in these studies is the incorporation of full $(3,27)$, partial $(3,10)$, or non-weight-bearing positioning $(6,11,30)$. Weight bearing during a proprioceptive acuity test more closely approximates neural input from muscles and joints during functional activities, whereas the use of partial weight bearing may enable people with knee OA to complete a weight-bearing test while minimizing pain. In the MOST study, use of a non-weight-bearing measurement of JPS enabled collection of data on a large number of participants while avoiding a selection bias against those with joint pain. However, the measurement used may have less bearing on usual physical function. Therefore, it is possible that the lack of association between incident knee OA and JPS may have been due to the seated JPS measurement used in this study.

Although there are a variety of ways to assess proprioception, the majority of studies have shown proprioceptive acuity is impaired with older age $(3,18,30,32)$ and in patients with knee OA $(10,11,15,32,38)$. In the only longitudinal study involving the relationship between proprioception and knee joint worsening, impaired JPS was not associated with radiographic knee OA cross-sectionally or with incident or progressive radiographic OA longitudinally (6). Another longitudinal study that evaluated proprioceptive acuity and physical function (WOMAC and chair stand test) in individuals with knee OA found that the inaccuracy in sensing passive knee motion did not differ between individuals with good versus poor WOMAC outcome but was higher in individuals with poor compared with good chair stand outcome at the 3-yr follow-up (37).

Despite cross-sectional evidence that people with knee OA have impairments in both strength and proprioception (9-11,13,17,23,38,39), in the MOST cohort, it seems that 
knee extensor strength but not JPS predicts risk for incident symptomatic knee OA, and neither appears to be associated with incident radiographic knee OA. These findings do not preclude the possibility of relevance of the combination of strength and proprioceptive acuity to physical functional level. It indicates that quadriceps strength but not JPS, as measured in this study, is a protective factor for symptomatic knee OA. One would expect that knee extensor strength and proprioception are important for control of balance, and both of these as well as balance are impaired with knee OA (13). In fact, in an investigation of individuals with symptomatic knee OA, those with the combination of proprioceptive impairments and muscle weakness had greater functional limitations (defined by $100-\mathrm{m}$-walk and "getup-and-go" test times) than those with proprioceptive impairments alone (42). Therefore, future studies of the effects of proprioception might be directed toward better characterizing its role in predicting functional limitations or disability rather than knee pathology.

Data from this study should be interpreted in light of the following considerations. In the present study, both the isokinetic strength and the non-weight-bearing JPS measures assessed different sensorimotor functions than those used in daily tasks. However, isokinetic dynamometry is a reliable measure and is feasible for this size study. Moreover, despite being non-weight bearing, our use of an active joint position test more closely approximated usual joint and muscle activity than a passive JPS test. The mean JPS in our study population $\left(4.2^{\circ}\right)$ is also similar to that found in other studies of individuals with knee OA and is not likely to have hindered our ability to detect a relationship $(2,11,13)$. In addition, the possibility of not being able to observe a relationship in this cohort of participants with risk factors for knee OA is unlikely due to muscle weakness of the cohort because there was sufficient variability within the range of strength data $(22-270 \mathrm{~N} \cdot \mathrm{m}$ in men and $20-206 \mathrm{~N} \cdot \mathrm{m}$ in women) and the rate of incident radiographic knee OA in our study population $(\sim 7 \%)$ during $2.5 \mathrm{yr}$ is similar to that found in previous reports $(4,8)$. For the study of incident radiographic tibiofemoral knee OA, we used the KL grading system for knee radiographs, and it is possible that a more sensitive measure of knee structure, such as magnetic resonance imaging, or a longer study duration may have led to the identification of a larger number of cases.

\section{REFERENCES}

1. Bennell KL, Hinman RS, Metcalf BR. Association of sensorimotor function with knee joint kinematics during locomotion in knee osteoarthritis. Am J Phys Med Rehabil. 2004;83(6):455-63; quiz 464-6.

2. Bennell KL, Hinman RS, Metcalf BR, et al. Relationship of knee joint proprioception to pain and disability in individuals with knee osteoarthritis. J Orthop Res. 2003;21(5):792-7.

3. Bullock-Saxton JE, Wong WJ, Hogan N. The influence of age on weight-bearing joint reposition sense of the knee. Exp Brain Res. 2001;136(3):400-6.

4. Cooper C, Snow S, McAlindon TE, et al. Risk factors for the
In addition, this study included assessment of knee symptoms at baseline and 30-month follow-up, and it is possible that intermittent symptoms may not have been present at the time of the follow-up appointment. This would have biased results toward the null hypothesis. A potential limitation of our 30-month follow-up is that a longer duration may have led to the identification of a larger number of cases. However, the effect of strength on the outcomes would not necessarily be greater with a longer duration. Therefore, future studies could better characterize this relationship through more frequent strength measurements as well as a longer duration.

Although it may have been of interest to evaluate JPS in both knees, our study evaluated only right knees. However, this may not be a limitation because previous studies have shown that impaired proprioceptive acuity is generally present in both limbs $(10,23,38,42)$, the direction of our results did not differ between bilateral and right-side-only analyses, and sample sizes were sufficient to detect clinically meaningful associations. Lastly, the MOST cohort is composed of individuals with or at high risk for knee OA. Thus, results from this study are applicable to older adults with elevated risk for knee OA and are not necessarily generalizable to other groups.

In summary, our results suggest that individuals with greater knee extensor strength are at decreased risk for incident symptomatic but not radiographic knee OA regardless of JPS score. Because quadriceps weakness and reduced proprioceptive acuity are, in many cases, modifiable, future studies should also investigate whether sensorimotor dysfunction at the knee joint contributes to progression of knee OA.

This study was supported by National Institutes of Health grants to Boston University (David T. Felson, M.D.; AG18820), The University of lowa (James C. Torner, Ph.D.; AG18832), University of Alabama (Cora E. Lewis, M.D., M.S.P.H.; AG18947), University of California San Francisco (Michael Nevitt, Ph.D.; AG19069), and a Beeson Career Development Award (Neil Segal, M.D.; K23AG030945). The study sponsor had no role in the study design, data collection, analyses and interpretation of data, writing of the article, or submission of the article for publication.

The authors thank the participants and staff of the Multicenter Osteoarthritis (MOST) study.

The authors report no conflicts of interest.

Publication of the results of this study does not represent an endorsement by the American College of Sports Medicine.

incidence and progression of radiographic knee osteoarthritis Arthritis Rheum. 2000;43(5):995-1000.

5. Davis MA. Epidemiology of osteoarthritis. Clin Geriatr Med. 1988;4(2):241-55.

6. Felson DT, Gross KD, Nevitt MC, et al. The effects of impaired joint position sense on the development and progression of pain and structural damage in knee osteoarthritis. Arthritis Rheum. 2009;61(8):1070-6.

7. Felson DT, McAlindon TE, Anderson JJ, et al. Defining radiographic osteoarthritis for the whole knee. Osteoarthritis Cartilage. 1997;5(4):241-50. 
8. Felson DT, Zhang Y, Hannan MT, et al. The incidence and natural history of knee osteoarthritis in the elderly: the Framingham Osteoarthritis study. Arthritis Rheum. 1995;38(10): 1500-5.

9. Fisher NM, Pendergast DR. Reduced muscle function in patients with osteoarthritis. Scand J Rehabil Med. 1997;29(4):213-21.

10. Garsden LR, Bullock-Saxton JE. Joint reposition sense in subjects with unilateral osteoarthritis of the knee. Clin Rehabil. 1999; 13(2):148-55.

11. Hall MC, Mockett SP, Doherty M. Relative impact of radiographic osteoarthritis and pain on quadriceps strength, proprioception, static postural sway and lower limb function. Ann Rheum Dis. 2006;65(7):865-70.

12. Hassan BS, Doherty SA, Mockett S, Doherty M. Effect of pain reduction on postural sway, proprioception, and quadriceps strength in subjects with knee osteoarthritis. Ann Rheum Dis. 2002;61(5): 422-8.

13. Hassan BS, Mockett S, Doherty M. Static postural sway, proprioception, and maximal voluntary quadriceps contraction in patients with knee osteoarthritis and normal control subjects. Ann Rheum Dis. 2001;60(6):612-8.

14. Herzog W, Longino D, Clark A. The role of muscles in joint adaptation and degeneration. Langenbecks Arch Surg. 2003; 388(5):305-15.

15. Hortobagyi T, Garry J, Holbert D, Devita P. Aberrations in the control of quadriceps muscle force in patients with knee osteoarthritis. Arthritis Rheum. 2004;51(4):562-9.

16. Hurley MV. Evaluation of reliability, reproducibility and validity of two methods of assessing proprioceptive acuity in the lower limb. Br J Rheumatol. 1996;35(1 suppl):140.

17. Hurley MV. The role of muscle weakness in the pathogenesis of osteoarthritis. Rheum Dis Clin North Am. 1999;25(2):283-98.

18. Hurley MV, Rees J, Newham DJ. Quadriceps function, proprioceptive acuity and functional performance in healthy young, middleaged and elderly subjects. Age Ageing. 1998;27(1):55-62.

19. Hurley MV, Scott DL, Rees J, Newham DJ. Sensorimotor changes and functional performance in patients with knee osteoarthritis. Ann Rheum Dis. 1997;56(11):641-8.

20. Jefferson RJ, Collins JJ, Whittle MW, Radin EL, O'Connor JJ. The role of the quadriceps in controlling impulsive forces around heel strike. Proc Inst Mech Eng H. 1990;204(1):21-8.

21. Johnson SR, Archibald A, Davis AM, Badley E, Wright JG, Hawker GA. Is self-reported improvement in osteoarthritis pain and disability reflected in objective measures? J Rheumatol. 2007; 34(1):159-64.

22. Kellgren JH, Lawrence JS. Radiological assessment of osteoarthrosis. Ann Rheum Dis. 1957;16(4):494-502.

23. Koralewicz LM, Engh GA. Comparison of proprioception in arthritic and age-matched normal knees. J Bone Joint Surg Am. 2000;82(11):1582-8.

24. LaValley MP, McLaughlin S, Goggins J, Gale D, Nevitt MC, Felson DT. The lateral view radiograph for assessment of the tibiofemoral joint space in knee osteoarthritis: its reliability, sensitivity to change, and longitudinal validity. Arthritis Rheum. 2005;52(11):3542-7.

25. Ling SM, Fried LP, Garrett ES, Fan MY, Rantanen T, Bathon JM. Knee osteoarthritis compromises early mobility function: the Women's Health and Aging Study II. J Rheumatol. 2003;30(1): 114-20.
26. Lund H, Juul-Kristensen B, Hansen K, et al. Movement detection impaired in patients with knee osteoarthritis compared to healthy controls: a cross-sectional case-control study. J Musculoskelet Neuronal Interact. 2008;8(4):391-400.

27. Marks R, Quinney AH. Reliability and validity of the measurement of position sense in women with osteoarthritis of the knee. J Rheumatol. 1993;20(11):1919-24.

28. Marks R, Quinney HA, Wessel J. Proprioceptive sensibility in women with normal and osteoarthritic knee joints. Clin Rheumatol. $1993 ; 12(2): 170-5$.

29. Mikesky AE, Mazzuca SA, Brandt KD, Perkins SM, Damush T, Lane KA. Effects of strength training on the incidence and progression of knee osteoarthritis. Arthritis Rheum. 2006;55(5): 690-9.

30. Mohammadi F, Taghizadeh S, Ghaffarinejad F, Khorrami M, Sobhani S. Proprioception, dynamic balance and maximal quadriceps strength in females with knee osteoarthritis and normal control subjects. Int J Rheum Dis. 2008;11:39-44.

31. Nevitt MC, Peterfy C, Guermazi A, et al. Longitudinal performance evaluation and validation of fixed-flexion radiography of the knee for detection of joint space loss. Arthritis Rheum. 2007; 56(5):1512-20.

32. Pai YC, Rymer WZ, Chang RW, Sharma L. Effect of age and osteoarthritis on knee proprioception. Arthritis Rheum. 1997; 40(12):2260-5.

33. Radin EL, Yang KH, Riegger C, Kish VL, O'Connor JJ. Relationship between lower limb dynamics and knee joint pain. J Orthop Res. 1991;9(3):398-405.

34. Segal NA, Felson DT, Torner JC, et al. Greater trochanteric pain syndrome: epidemiology and associated factors. Arch Phys Med Rehabil. 2007;88(8):988-92.

35. Segal NA, Torner JC, Felson D, et al. Effect of thigh strength on incident radiographic and symptomatic knee osteoarthritis in a longitudinal cohort. Arthritis Rheum. 2009;61(9):1210-7.

36. Sharma L. Proprioceptive impairment in knee osteoarthritis Rheum Dis Clin North Am. 1999;25(2):299-314.

37. Sharma L, Cahue S, Song J, Hayes K, Pai YC, Dunlop D. Physical functioning over three years in knee osteoarthritis: role of psychosocial, local mechanical, and neuromuscular factors. Arthritis Rheum. 2003;48(12):3359-70.

38. Sharma L, Pai YC, Holtkamp K, Rymer WZ. Is knee joint proprioception worse in the arthritic knee versus the unaffected knee in unilateral knee osteoarthritis? Arthritis Rheum. 1997; 40(8):1518-25.

39. Slemenda C, Brandt KD, Heilman DK, et al. Quadriceps weakness and osteoarthritis of the knee. Ann Intern Med. 1997; 127(2):97-104.

40. Slemenda C, Heilman DK, Brandt KD, et al. Reduced quadriceps strength relative to body weight: a risk factor for knee osteoarthritis in women? Arthritis Rheum. 1998;41(11):1951-9.

41. Solomonow M, Krogsgaard M. Sensorimotor control of knee stability. A review. Scand J Med Sci Sports. 2001;11(2):64-80.

42. van der Esch M, Steultjens M, Harlaar J, Knol D, Lems W, Dekker J. Joint proprioception, muscle strength, and functional ability in patients with osteoarthritis of the knee. Arthritis Rheum. 2007;57(5):787-93.

43. Washburn RA, Smith KW, Jette AM, Janney CA. The Physical Activity Scale for the Elderly (PASE): development and evaluation. J Clin Epidemiol. 1993;46(2):153-62. 